\title{
Sri Lankan Perspectives on Pharmacist Administered Vaccinations
}

\section{Bushell MJA ${ }^{1,3^{*}}$, Morrissey $\mathrm{H}^{1,3}$, Waidyarathne $\mathrm{E}^{2}$, Zawahir $\mathrm{S}^{2}$ and Ball $\mathbf{P}^{1,3}$}

${ }^{1}$ Charles Darwin University, Darwin Northern Territory, Australia

${ }^{2}$ University of Ruhuna, Galle, Sri Lanka

${ }^{3}$ Pharmacy Discipline, School of Psychological and Clinical Sciences, Faculty of Engineering, Health, Science and Environment, Charles Darwin University Casuarina Campus, Darwin

\section{Abstract}

Background: Globally, the administration of vaccines by pharmacists is gaining momentum and incorporation of vaccination and injection skills in pharmacy school curricula is becoming more common.

Objectives: This paper reports on one of four focus groups conducted to validate an Australian developed undergraduate vaccination training program (VTP) and its wider application beyond the Australian health care environment.

Methods: A focus group discussion was conducted with six Sri Lankan medical and pharmacist experts following the development of a vaccination training program.

Results: Participants voiced that prior to incorporation of the VTP into undergraduate Sri Lankan curricula, approval should be sought from the Ministry of Health and established vaccinators. The issue of certificates of competency was deemed inappropriate to be issued to Sri Lankan students. Additionally the assessment of competency was seen to be essential on a yearly basis throughout the curricula. Concern was expressed concerning the management of vaccine associated anaphylaxis in the context of minimal pre-hospital care. Participants voiced that vaccination training should be piloted prior to formal incorporation into Sri Lankan undergraduate pharmacy curricula.

Conclusion: While there is wide interest in the introduction of clinical skills into pharmacy curricula, the results of this study raise questions about the inclusion of injection and vaccination training in Sri Lankan undergraduate curricula.

Keywords: Focus groups; Vaccination; Sri Lanka; Pharmacy practice; Clinical pharmacy

Abbreviation's: PAVs: Pharmacist Administered Vaccinations; SLMC: Sri Lanka Medical Council; MBBS: Bachelor of Medicine and Bachelor of Surgery; CPD: Continuing Professional Development; VTP: Vaccination Training Program

\section{Introduction}

In many countries including, the United States of America (USA) and Canada, administration of vaccines by pharmacists is an established role, while in other countries, including Australia and New Zealand [1], it is an emergent role and in other countries the vaccination service delivery by pharmacists remains a foreign concept. In Sri Lanka the role of the pharmacist is evolving, however it does not currently include the administration of vaccines.

The title 'pharmacist' in Sri Lanka may at present be applied to a person who has completed a two year certificate of proficiency in pharmacy, or a two year certificate of efficiency in pharmacy, or a four year bachelor degree either as a two year Bachelor of Science followed by two year specialisation in Pharmacy or as the four year Bachelor of Pharmacy. In Sri Lanka, registrations of pharmacists who fulfil the prescribed requirements are registered by the Sri Lanka Medical Council (SLMC) [2].

Currently the majority of pharmacists hold the proficiency or efficiency certificate in pharmacy, as the first Bachelor of Pharmacy degree was established in 2006 [3]. The certificate system had previously satisfied Sri Lanka's needs however with the emerging changes in pharmacists' role around the world particularly in clinical pharmacy; Sri Lanka wants to expand the knowledge and skill base of future pharmacists and is also moving towards the degree program. It was made clear from the outset, that such expended roles for pharmacists were only being considered for those with a four year qualification. Field experience or placements for real life training is now incorporated into Sri Lankan undergraduate curricula bringing them in line with mainstream pharmacy education standards. One element still in gestation is clinical pharmacy; both education and practice, however there is a strong intention to integrate aspects of clinical pharmacy into the Sri Lankan pharmacy undergraduate curriculum [3]. Currently there is no compulsory internship year, prior to practicing as a registered or listed pharmacist.

Sri Lanka has highly developed medical education and many medical staffs have worked in countries where clinical pharmacy services are well-developed. They can see the value and support the concept to expand the role of the pharmacist to include clinical services. Administration of vaccines by pharmacists has been considered as a clinical skill that undergraduate pharmacists can learn, but the idea of actual practice of vaccinations after graduation is not yet established.

At present the majority of nurses practicing in Sri Lanka are hospital trained; two cohorts have graduated from a newly established Bachelor of Nursing Degree. Thus, historically nurses' responsibilities rarely extend beyond traditional patient care $[4,5]$. Currently, nurse administered vaccinations must be done under the direct supervision of a medical doctor.

Medical education in Sri Lanka is consistent with the education requirements of medical students in most countries. Students must successfully complete a five-year undergraduate Bachelor of Medicine

*Corresponding author: Bushell MJA, Charles Darwin University, Darwin Northern Territory, Australia, Tel: 088946 7490; E-mail: mary.bushell@cdu.edu.au

Received January 19, 2015; Accepted February 24, 2015; Published March 03 2015

Citation: Bushell MJA, Morrissey H, Waidyarathne E, Zawahir S, Ball P (2015) Sr Lankan Perspectives on Pharmacist Administered Vaccinations. Pharmaceut Reg Affairs 4: 135. doi:10.4172/2167-7689.1000135

Copyright: (C) 2015 Bushell MJA, et al. This is an open-access article distributed under the terms of the Creative Commons Attribution License, which permits unrestricted use, distribution, and reproduction in any medium, provided the original author and source are credited. 
and Bachelor of Surgery (MBBS), followed by an internship and supervised practice. Doctors must continue to maintain currency of practice completing mandatory Continuing Professional Development (CPD). Physicians must also undertake specialisation before the age of 35 , or remain as general practitioners (which is in itself a specialisation). Medical academics are permitted to continue practice while they are in an academic teaching role.

Pre-hospital care is another emerging concept in Sri Lanka and most ambulance services offer simple patient transport with first aid, but no advanced trauma equipped ambulance vehicles are currently in service, and they do not carry paramedics.

The Ministry of Health is the decision making authority for all immunisation related activities in the country [6]. All vaccinations should be administered at centres which are registered and accredited by the Ministry of Health. Vaccinations are currently offered via immunisation clinics under the supervision of Medical Officer of Health $(\mathrm{MOH})$, both doctors and trained Public Health Inspectors or Government Health Midwives may administer vaccinations [7]. Some, vaccinations are offered in the government and private hospital setting. In the private hospital such vaccinations are offered at patient request, the private hospital caters for caters to $5-10 \%$ of the country's in-patients [8]. In government hospitals vaccinations are most often offered when a person has a pre-existing condition that requires hospital access or have an injury that requires for example, a tetanus vaccine. Vaccination offered at government hospitals and ministry of health immunisation clinics are free of charge.

Collaboration between the pharmacy disciplines at Charles Darwin University and the University of Ruhuna was established in 2014 and this facilitated the formation of a focus group to discuss and validate a pharmacy undergraduate vaccination program that can be jointly introduced as a new clinical skill for pharmacy students through a pilot study in both universities.

\section{Aim}

This paper reports on one of four focus groups conducted to validate an Australian developed undergraduate vaccination training program (VTP) and its broader application outside the Australian health care environment.

\section{Method}

The study adopted a mixed descriptive and explorative method in innovation of teaching, through number of activities that include development of training module material, multidisciplinary focus groups to validate this module, then evaluation of the achieved students attributes by students and multidisciplinary practitioners. The part discussed in this paper is the outcome of one of the four conducted focus groups, which was conducted in Sri Lanka. Focus groups were chosen to assist in the validation of the Australian developed undergraduate pharmacy specific vaccination training program, as they allow a panel of multidiscipline experts to voice their concerns, feedback, ideas and views on the content and the wider usability of the educational material and modes of delivery. A total of six participants were recruited. Criteria for inclusion included having an extensive knowledge in vaccination administration and a medical or pharmacy qualification. It was intended to have a similar expertise composition to that of the two focus groups conducted in Australia and the USA. Participants included the manager of a large pharmaceutical corporation (pharmacist), a consultant physician (hospital practice), two senior medical academics (one a general practitioner), one practicing general practitioner and a senior pharmacist academic. Participants were electronically sent a copy of a plain language statement outlining the study and a consent form. Individual consent forms were signed by each participant prior to the commencement of the focus group. No difficulties were experienced with recruitment; supporting the assertion that there is wide interest in the introduction of clinical skills into undergraduate pharmacy curricula in Sri Lanka.

The focus group was steered by a moderator, the developer of the vaccination training program. The primary responsibility of the moderator was to encourage open discussion among participants and keep the discussion on track, without having influence into the decision making.

Ten open-ended guide questions had been developed and were asked in a predetermined order. The focus group meeting was audio recorded (with consent from all participants), transcribed verbatim, and then manually analysed for themes. In addition, each guide question was published on an A3 laminated poster and participants were provided with colour coded self-adhesive note pads (sticky notes). Participants were encouraged to write any thoughts on the provided sticky note pads and then asked to attach the written note to the corresponding guide question during the discussion. Statements and feedback written by participants was then analysed for themes alongside the audio recording.

Note was taken of whether emerging and identified themes were a consensus among participants or if themes were the view point of one or two participants. Apparent factions and group disparities were manually coded. Coding was validated by the project supervisor.

The focus group session ran for 170 minutes, with 10 minutes for opening and 5 minutes for closing remarks and one 30 minute break.

\section{Results}

\section{The analysis revealed four themes}

Approvals prior to incorporation of vaccination training into Sri Lankan curriculum, gaining approval and the incorporation of any vaccination training program into Sri Lankan undergraduate pharmacy curricula was a dominant theme and several subthemes could be identified.

\section{Approval by the ministry of health}

This was the most-discussed topic. It was obvious that participants were in agreement that support for the inclusion of the clinical skill into pharmacy curricula is required. The general rule and practice to introduce any new module into an existing curriculum is that the new module should be submitted with justification at the university's faculty curriculum development and evaluation committee and then subsequently forwarded to University council for approval via the relevant faculty board. However, practicing these skills in the future is still a question for the Ministry of Health as currently as administration of vaccination by pharmacists is not currently practiced in Sri Lanka. Additionally, if the clinical service is to be introduced, it will have implications on a number of professional boundaries, graduate supply and other resource implications. "You should not do anything without government approval, I think if not it will create problems, I think the education is a basic concept, but if you are going to teach these students to provide this service for the country you need minister approval" Physician 1. "Before implemented in Sri Lanka it needs to be addressed in government policies before it is introduced into our system" Physician 2. 
While such comments were articulated by physicians there was universal consensus around the table that there was a need for approval from external regulatory bodies for inclusion of any vaccination training program or injection skills outside the pilot.

\section{Approval from established vaccinators}

Participants reported that endorsement by established vaccinators was important, which in this case physicians or nurses under direct supervision of a physician, prior to inclusion into the undergraduate curricula. The skill was recognised as being new to pharmacy students and the pharmacy profession and therefore other professionals needed to be informed.

"If you incorporate this thing in Sri Lanka, with competency after four years, other doctors, other professionals need to be advised" Physician 1.

"It is not incorporated into any other B. Pharm in Sri Lanka so other health professionals need to know" Pharmacist $A$

\section{Assessment and recognition of competency}

Integrated and repeated assessment: Participants expressed that assessment must be repeated and ongoing; however initial delivery of the skills would be preferred as a one week intensive teaching session in one identified module.

"One intensive week course-I personally feel is better in our set up as the curriculum is already full, however assessment wise, skills, there should be something put in regularly, even in second year, otherwise they will forget everything" Physician 3.

\section{Certificate of competency}

Participants expressed concern about the issuing of a certificate of competency in the skills of injection. Both physician and pharmacist participants mentioned this would be interpreted as being qualified to administer injections, such as penicillin and other antibiotics. There was a uniform consensus that a certificate of competency should not be issued to students.

"By offering a certificate of competency you are offering a pass out and pharmacy students will start vaccinating" Physician 4.

\section{Pilot training program and recognition of the Sri Lankan context}

The researchers detected some hesitancy about the delivery of the vaccination training program as a pilot in Sri Lanka from some medical doctor participants. However verbally the group was in agreement that the training program needs to be conducted as a pilot program initially, possibly on a number of occasions and preferably as multidisciplinary offering to include medical and nursing students in addition to pharmacy students and then evaluated prior to incorporation into the curricula.

"Before incorporating it into the curriculum can it be ran as a trail" Physician 2.

A recurring theme throughout the focus group was the need for the developed vaccination training program to recognise the unique characteristics of Sri Lanka's health care system, the diverse pharmacists' and nursing qualifications, the patterns of health demand; and the manual prescribing, dispensing and health records.

"Our set up is completely different" Physician 3.

The Sri Lankan focus group also identified that the topics in modules should be separated as chapters and that pharmacists should be competent in their knowledge about all vaccines not only those they are allowed to administer. Additionally the group recommended the delivery of the material through-out the four years of the pharmacy course not only three and an additional competency assessment annually thereafter if the student vaccinator did not perform a certain number of actual vaccinations. Additionally the current Bachelor Degrees in Sri Lanka are accredited under total credit points as in Australia, the pharmacy course using all allowable credit point for other theory and foundation knowledge units and it will be required to shift or replace some topics to allow the inclusion of the vaccination modules.

\section{Role of pharmacists in anaphylaxis}

Medical participants voiced concerns about the critical nature of anaphylaxis. Many participants expressed concern about the ability of the pharmacist to appropriately respond to the medical emergency anaphylaxis for many reasons:

- The diverse qualifications of pharmacists

- The current community pharmacies' floor plans and size

- The inability to have an ambulance dedicated to the place where vaccination will be conducted

- The inability to have the vaccinated pharmacist directly supervised by physician as is currently the case with vaccinating nurses

- The lack of pre-hospital care

This concern was amplified by a case report.

"Sri Lanka we have problems there was a case one or two years back, fatality from anaphylaxis" Physician 1

Participants appeared relieved when it was articulated that it was not currently under consideration that pharmacists would administer antibiotics such as penicillin, a medication more strongly correlated with a prevalence of inducing anaphylaxis.

"Are you planning for pharmacists to administer other medications?" Physician 2, participant interjects "antibiotics should not be administered by pharmacists" Physician 1 .

It was recognised that administering adrenaline in case of anaphylaxis is considered as an injection other than vaccination, however this will be the only medication allowed to be administered by pharmacists and its administration is included in the undergraduate training program.

\section{Discussion}

As the role of the pharmacist expands in Australia to embrace this patient care service it is integral that pharmacy students, like any other health professionals, are educated and deemed competent to safely and appropriately administer vaccines [9]. Such competence should be achieved via successful completion of a validated vaccination training program. Focus group discussion was used to explore the newly developed training program and gather perspectives on the usability for both students studying a Bachelor of Pharmacy in and outside Australia. This article attempts to answer the research question on the global usability of the newly developed vaccination training program. Themes that arose that are discussed here include: 
- Approvals prior to incorporation into Sri Lankan curricula

- Assessment of competency

- Piloting and contextualisation of content

- Role of pharmacists in anaphylaxis

The group interaction facilitated participants to develop and define their beliefs voicing their positions in presence of others from different disciplines as they explained their view points.

This focus group was the final focus group in a series of four, the first focus group being held in USA and the second and third held in Australia. It was felt there was value in reporting on this focus group separately, in addition to combined with the other three, for several reasons. Pharmacy student vaccination training and pharmacist administered vaccination are well-established and accepted concepts in the USA. However, while it is not yet an established practice in Australia, the acceptance of pharmacist administered vaccinations is gaining momentum as jurisdictional regulations are rapidly being amended to enable pharmacists to administer vaccines. Further, it has been identified by the Pharmacy Board of Australia that the administration of vaccines is within the scope of the Australian pharmacist practice. Thus participants involved in the Australian and the USA focus groups were more accepting of the concept shifting their focus to the practicality and applicability of the content of the developed vaccination training program for undergraduate pharmacy students rather than the novelty of the concept of pharmacists vaccinating. On the otherhand, the Sri Lankan focus group provided great benefit to validating the concept of pharmacists vaccinating under all other health services environments and constrains that were not previously considered and further validation on the developed vaccination training program and its use ability and incorporation outside of Australia into Sri Lankan undergraduate curricula.

\section{Conclusion}

Vaccination is a new skill for a pharmacist that is currently under investigation for the very near future inclusion into the clinical pharmacy practice in Australia. It has been identified that Sri Lankan health professionals and academics see value in incorporating clinical skills into their newly established undergraduate Bachelor of Pharmacy programs. Vaccination is one such clinical skill that is being considered. However prior to the introduction of vaccination and injection skills training in Sri Lanka, approval and endorsement external to university pharmacy schools should be sought. This focus group identified that the Ministry of Health should be consulted and government policies modified, further representatives from established vaccinator professions should be informed. Such consultation and collaboration would provide a foundation for successful introduction of the skill of vaccination to be taught in undergraduate pharmacy curricula, contributing to pharmacy profession readiness should the clinical skill be incorporated into the practice scope of pharmacists in Sri Lanka.

\section{Acknowledgement}

Participants in the focus group and the pharmacy staff at the University of Ruhuna, Sri Lanka.

\section{References}

1. Bushell MJA, Yee KC, Ball PA (2013) Case for pharmacist administered vaccinations in Australia. Journal of Pharmacy Practice and Research 43: 292.

2. Sri Lanka Medical Council.

3. Coombes I, Fernando G, Wickramaratne M, Peters N, Lynch C, et al. (2013) Collaborating to develop clinical pharmacy teaching in Sri Lanka. Pharmacy Education 13: 29-35.

4. Jayasekara RS (2009) Issues, challenges and vision for the future of the nursing profession in Sri Lanka: a review. International Nursing Review 56: 21-27.

5. De Silva BSS, Rolls C (2010) Health-care system and nursing in Sri Lanka: An ethnography study. Nursing and Health Sciences 12: 33-38.

6. Epidemiology Unit Ministry of Health (2012) Surveillance of Adverse Events Following Immunization. Ministry of Health, Sri Lanka.

7. Ministry of Health: Government of the Democratic Socialist Republic of Sr Lanka. Comprehensive multi-year plan for immunisation, 2012-2016.

8. RAM. Standpoint Commentary: The Private Healthcare Sector of Sri Lanka.

9. Hope D, Haywood A, Bernaitis N (2014) Current research: Incorporating vaccine administration in pharmacy curriculum: Preparing students for emerging roles. Australian Journal of Pharmacy 95: 60-63. 